\title{
ALGUNOS PROBLEMAS EN LA ENSEÑNZA DE LA RELATIVIDAD
}

\author{
PÉREZ, H. ${ }^{1}$ y SOLBES, J. ${ }^{2}$ \\ ${ }^{1}$ IES de Sedaví, Valencia \\ 2 IES J. Rodrigo Botet. Manises \\ jordi.solbes@uv.es
}

\begin{abstract}
Resumen. La relatividad es una parte importante de la física moderna. En este artículo se analiza su enseñanza-aprendizaje en la enseñanza secundaria y se muestran sus principales dificultades. El artículo argumenta razonadamente contra prácticas tradicionales en la enseñanza de la relatividad y finalmente apunta una nueva aproximación a su enseñanza.
\end{abstract} Palabras clave. Enseñanza-aprendizaje, relatividad, didáctica de la física.

Summary. Relativity is a very important part of modern physics. In this paper we analyze the teaching and learning of the theory of relativity at secondary school level and we show the pupils' main difficulties. This article presents some reasoned arguments against traditional methods to teach relativity. Finally, a new approach to relativity teaching is showed.

Keywords. Teaching and learning, theory of relativity, teaching of physics.

\section{INTRODUCCIÓN}

\section{Planteamiento del problema}

La enseñanza de la física moderna en el anterior bachillerato, en el cual no se introducía la relatividad, sino tan sólo las relaciones masa-energía en la física nuclear, aparecía ya como problemática a comienzos de los ochenta. Gil, Senent y Solbes (1986), en un amplio estudio acerca de la introducción de la física moderna en la secundaria, constatan que la enseñanza de los aspectos más modernos de la física ha estado habitualmente relegada de los planes de estudio y que ésta se realiza de una forma poco estructurada, que no deja clara la existencia de dificultades insuperables que originaron la crisis de la física clásica y no diferencia entre ambas ni señala los límites de validez de la clásica. Señalan abundantes errores conceptuales en textos y concluyen que los alumnos no logran una mínima comprensión de los conceptos e ideas de la física moderna. Propugnan una aproximación que parta de la crisis de las concepciones clásicas y muestre cualitativamente las caracte- rísticas del nuevo paradigma (Gil y Solbes, 1993). Estos problemas eran similares a los detectados en otros países (Hewson 1982; Villani y Pacca, 1987).

Por todo ello, en las últimas décadas se ha originado un movimiento de renovación para introducirla, entre cuyas razones podemos destacar:

1) Proporciona una visión más correcta de cómo se desarrolla la ciencia, evitando visiones lineales, acumulativas y mostrando cómo la física clásica no pudo explicar los problemas que se suscitaban, lo que provocó su crisis y el desarrollo de la física moderna.

2) La importancia de la física moderna en la sociedad no sólo por sus desarrollos tecnológicos, sino por su influencia en el pensamiento y la cultura de su tiempo. 
3) El interés y la curiosidad que manifiestan los alumnos por dichos desarrollos y por cuestiones más teóricas; por ejemplo, en el caso de la relatividad, la paradoja de los gemelos, la simultaneidad o los agujeros negros.

4) Porque la enseñanza de la física moderna mejora la comprensión de la propia física clásica, al mostrar sus límites de validez y las diferencias entre ambas.

En resumen, porque proporciona al alumno referencias de la cultura científica actual. En esa línea argumentan recientemente otros autores como Osterman y Moreira (2000) o Shabajee y Postlethwaite (2000).

Otras razones que justifican más específicamente la introducción de la relatividad son las siguientes:

1) Porque facilita la comprensión de los conceptos de espacio, tiempo y energía (Tarín, 2000), ya que con el estudio de la relatividad se ofrece al alumnado la posibilidad de reexaminar críticamente conceptos básicos, que fueron introducidos en etapas más tempranas.

2) Su interés como teoría física fundamental que, junto con la física cuántica, proporciona el marco en el interior del cual deben ser elaboradas las nuevas teorías (toda teoría fundamental debe ser invariante bajo las transformaciones de Lorentz y debe estar cuantizada (Sánchez Ron, 1985).

Así pues, parece positivo para la formación científica de los estudiantes que se haya incluido la teoría de la relatividad en los nuevos currículos de física del bachillerato LOGSE. Esto supone un reto didáctico para los profesores. En efecto, si bien el afán por divulgar y enseñar los fundamentos de la teoría goza de una amplia tradición histórica en las exposiciones elementales de la relatividad, iniciada ya por el propio Einstein, lo cierto es que no se le ha prestado, en cambio, una atención paralela a su didáctica, especialmente en la enseñanza secundaria. Además, en los últimos años, se han abierto interesantes debates y perspectivas que rompen la uniformidad de los tratamientos tradicionales.

Estas breves referencias apuntan claramente a la existencia de problemas didácticos, que este trabajo intentará clarificar. En concreto:

1) ¿Cómo se introduce en la enseñanza secundaria la relatividad y sus prerrequisitos tanto desde el punto de vista científico cómo didáctico?

2) ¿Son comprendidos estos conceptos por los alumnos? ¿Qué dificultades encuentran?

\section{FORMULACIÓN DE HIPÓTESIS. FUNDA- MENTACIÓN}

De acuerdo con lo anterior se formula la siguiente hipótesis:

Los conceptos de tiempo, espacio y sus propiedades, así como los distintos sistemas de referencia, se introducen ya desde los niveles inferiores de la secundaria de forma inconexa, acrítica y poco reflexiva. La enseñanza de la teoría de la relatividad se realiza de forma poco clarificadora, sin tener en cuenta las preconcepciones de los alumnos, y sin resaltar su posición en la estructura de la física. En consecuencia, el aprendizaje es escasamente significativo y no se consolidan las nuevas concepciones en los estudiantes.

La fundamentación de la hipótesis toma como base teórica la didáctica de las ciencias, que configura un cuerpo teórico organizado en torno a un consenso básico: la orientación constructivista. A continuación se recogen los rasgos más importantes que de la enseñanza de la relatividad ha dibujado la investigación didáctica, punto de partida que ha de orientar este trabajo.

Una parte sustancial de los estudios han girado en torno a las estrategias de cambio conceptual. Se ha utilizado incluso con carácter de ejemplo para contrastar modelos (Hewson, 1982), pues la teoría de la relatividad supone una formulación nueva de los conceptos clásicos de espacio y tiempo que son concepciones muy potentes, arraigadas en la mente y la psicología humana, tanto que fueron considerados como categorías a priori de la sensibilidad por Kant.

Todos los esfuerzos en los niveles anteriores se han dirigido a consolidar la concepción clásica, modificando los esquemas espontáneos. La tarea no es sencilla y numerosos trabajos han mostrado la presencia de potentes obstáculos en la construcción y consolidación del modelo newtoniano. Lo que se está solicitando, con la relatividad, al estudiante es la superación del mismo. Según Colombo (1995): «A pesar de estas limitaciones, en contraposición con los sistemas conceptuales de sentido común (poco estructurados, con pobre interrelación y gran incoherencia), un sistema científico tiene una estructura mucho más consolidada, con fuertes interconexiones, de gran validez y consistencia... ¿No cabría esperar, entonces, que, desde un punto de vista cognoscitivo, un paradigma científico sea aún más difícil de modificar que los espontáneos?»

En el caso de la relatividad, tal y como resaltan Toledo y otros (1997): «Los alumnos se encuentran ante una situación nueva frente a la física clásica y no pueden acudir a experiencias cotidianas para aceptar la plausibilidad de la teoría o para corroborar la eficiencia de la misma.» En el transcurso de su trabajo muestran que tras una instrucción formal: a) los nuevos conceptos de la relatividad espacial no han desplazado a los anteriores de la física clásica, sino que se han unido a ellos en una interacción compleja; $b$ ) el aprendizaje de contenidos específicos es más bien superficial, sin anclajes firmes que permitan resolver situaciones fuera de aquéllas desarrolladas en la instrucción formal.

Conclusiones análogas encontramos en otros autores (Villani y Arruda, 1998; Alemañ, 2000), que incluso renuncian explícitamente a considerar que se ha dado un autentico cambio. Un problema adicional relevante es la persistencia y estabilidad de las concepciones relativistas, que, como se ha mostrado, es muy baja (Gil y Solbes, 1993; 
Villani y Pacca, 1987), de tal forma que estudiantes que mostraron un conocimiento aplicado de los principios, situados al cabo de un tiempo ante análisis simples, recurren a nociones que involucran conceptos de espacio y tiempos absolutos. Incluso estudiantes avanzados usan nociones espontáneas en la solución de problemas acerca de la velocidad de la luz, próximas a otras señaladas en el campo de la cinemática por otros autores.

A continuación se repasan algunos aspectos problemáticos, pero vaya por delante la salvedad de que en este campo de la relatividad, y en general de la física moderna, las ideas espontáneas o intuitivas no han sido cabalmente estudiadas. Las principales líneas de pensamiento de los alumnos han sido potenciadas por la enseñanza previa, los medios de comunicación y el uso deformado de los aspectos llamativos de la teoría en la cultura popular y la divulgación

En palabras de Mach, citado por Williams (1968), «... precisamente los principios mecánicos de apariencia más simple son de un carácter sumamente complicado»». La investigación acerca de estos y otros conceptos, como los de sistema de referencia, principio de relatividad de Galileo, masa inercial y gravitatoria, etc. adquiere una importancia primordial para nuestros propósitos, ya que son el punto de partida de la introducción de la teoría de la relatividad.

\section{Ideas acerca de los conceptos de espacio y tiempo}

Desde una perspectiva didáctica se han estudiado aspectos parciales del problema. El espacio entre dos sucesos es considerado por los alumnos como universal, conforme a la formulación newtoniana: «una visión espontánea que considera a las distancias en ellas mismas independientes de los observadores» (Villani y Pacca, 1987). Sin embargo las concepciones que mantienen no son iguales a las que se han acuñado en la tradicional visión newtoniana; atributos de esta visión, tales como continuidad, homogeneidad, isotropía, etc., distan mucho de ser asumidos por los alumnos. El concepto de continuo en su aspecto geométrico-matemático ha sido estudiado por Romero (1996), quien concluye que «desde el punto de vista didáctico la hipótesis de intuitividad inmediata del continuo no tiene base que la sostenga» e insiste en que "en el caso de los alumnos de nuestra muestra el esquema conceptual del continuo es un agregado inconexo de imágenes». La isotropía tampoco reviste la sencillez que se le podría presuponer. Investigaciones sobre el concepto, como propiedad de los materiales, con alumnos de primer ciclo universitario muestra su deficiente asimilación (Gallegos, 1992).

El estudiante no razona en términos de propiedades del espacio-tiempo, sino que mantiene una visión mecanicista que requiere de propiedades fijas y cuerpos extensos para una visión realista de la naturaleza. Como expone Hewson, (1982) esto plantea un problema, pues el alumno asume como valor real únicamente lo que está en reposo respecto a él. Si de lo que se trata es de medir algo en movimiento, por ejemplo, la duración de un fenómeno como la vida de una partícula inestable o una longitud, el valor obtenido no posee el mismo estatus de realidad: admite que «parece» que la longitud y el tiempo son diferentes, pero «en realidad» son absolutos y hay un único valor real.

\section{Acerca del principio de relatividad}

Ya desde los niveles inferiores comprender el principio de relatividad galileana plantea graves dificultades: «El problema de la relatividad del movimiento es muy difícil para estos alumnos ( $4^{\circ}$ de ESO). Analizar el movimiento desde un sistema de referencia exterior a uno mismo es algo que se consigue sólo en un estado de desarrollo mental avanzado y con bastante entrenamiento.» (Hierrezuelo, 1993). Esto incide en las dificultades en el manejo de los prerrequisitos con que se inicia el aprendizaje de la relatividad.

En cuanto a la relatividad einsteniana, Villani y Pacca (1987) han mostrado cómo incluso estudiantes universitarios, en cuestiones que involucran la propagación de la luz y a varios observadores, manejan la idea de movimiento absoluto y nociones que parecen moverse entre un plano de lo real y un plano «aparente», tal como se ha indicado en el apartado anterior. Concluyen afirmando que no es realista partir de la base de que los alumnos poseen un completo conocimiento del principio clásico de relatividad de Galileo y propugnan que, en la introducción a la relatividad einsteniana, el trabajo de construir la intuición relativista pase primero por la construcción de la intuición galileana que libere a los estudiantes del espacio absoluto.

\section{Acerca del marco de referencia}

En mecánica, la definición de sistema de referencia inercial (SRI) está asociada a la primera ley de Newton. Dicha ley no cabe introducirla como caso particular de la segunda, so pena de que se quiera utilizar el concepto de sistema de referencia absoluto y caer en un razonamiento circular. Éste es el significado profundo de la primera ley «y no seguir este tratamiento en la escuela impide la compresión de los alumnos». De hecho, el estudio del «principio de relatividad Galileo-Newton ayuda a una mejor transición a la relatividad einsteniana» (Parasnis, 1998).

En cuanto a las concepciones de los alumnos respecto a los marcos de referencia newtonianos, Saltiel y Malgrange (1980) indican: a) La velocidad, la distancia recorrida en un movimiento y la trayectoria de un objeto en movimiento son vistas como independientes del marco de referencia. b) El movimiento es explicado descriptivamente con relación a un espacio absoluto; en este esquema hay una sola velocidad verdadera y un verdadero espacio atravesado, y las diferencias en las medidas obtenidas por varios observadores en movimiento son causadas por movimientos aparentes. c) En situaciones de movimientos «arrastrados»» se usa la idea de adición de movimientos absolutos en vez de las transformaciones de velocidades en el movimiento relativo. Esto es indicativo de las dificultades del punto de partida en que las nociones clásicas no han sido asumidas en su complejidad. 
Consecuencia directa de esto para el aprendizaje de la relatividad especial es la presencia de observadores privilegiados que perciben los valores «reales». La valoración de lo que es real o no está siempre presente. Los estudiantes admiten que diversos observadores «parece» que obtienen diversas medidas, pero en «realidad» hay sólo una medida del espacio, el tiempo o la velocidad de la luz. Estas ideas son recogidas por varios autores (Hewson 1982, Villani y Pacca, 1987).

\section{Acerca de la velocidad de la luz}

Otro trabajo con estudiantes universitarios (Villani y Arruda, 1998) ha puesto de manifiesto una gran resistencia a la aceptación de los principios de la teoría. En concreto, los estudiantes aceptan el carácter límite de la velocidad última de la luz (en un supuesto espacio absoluto implícito) y la composición galileana de velocidades siempre que el resultado no exceda de $c$.

Por otra parte, la insistencia en la constancia de la velocidad de la luz, recogida en el segundo postulado de la relatividad, hace referencia al movimiento de la luz en el vacío y al problema de la determinación de su velocidad en distintos sistemas. Se ha de evitar así el error, frecuente en los alumnos, de suponer que esto se extiende a su paso por medios materiales. En todo caso cabe indicar que la velocidad por cualquier medio material en reposo o movimiento (problema del arrastre parcial de Fizeau) es inferior a $c$.

En términos generales no parece que en el aprendizaje de la cinemática o la óptica se aborde, aunque sea tangencialmente, el problema de la medición de la velocidad de la luz y su relevancia lógica y epistemológica.

\section{Ideas acerca de la masa}

Las ideas manejadas por los estudiantes acerca de la masa son diversas y usadas según el contexto: masa como cantidad de materia, principio de conservación de la masa (en la química elemental), masa variable con la velocidad (inducida por la ciencia-ficción, la divulgación, etc.). Parasnis (1998) pone de manifiesto las dificultades que acarrea la definición de masa como cantidad de materia de un cuerpo, concepto escasamente definido.

El concepto de masa en el marco de la teoría de la relatividad ha suscitado un rico debate epistemológico y didáctico, cuyo detalle se puede encontrar en Solbes y otros (2002) y que aquí sintetizamos brevemente. A grandes rasgos se contrapone el uso de la masa invariante, como única masa, a la denominada masa relativista correspondiente a una partícula con masa en reposo $m$ que se mueve con velocidad $v$ y que se define como $m_{r}=m /\left(1-v^{2} / c^{2}\right)^{1 / 2}=\mathrm{rm}$. También se puede definir como $m_{r} \stackrel{r}{=} E / c^{2}$ siendo $E$ la energía total de la partícula. En este caso hay dos razones que propician el debate: en primer lugar, la posición del propio Einstein, que ha dado lugar a controversia (Adler, 1987; Carson, 1998); en segundo lugar, el hecho que la diferencia entre ambos puntos de vista sea de interpretación, dado que, evidentemente, la expresión de las leyes es la misma. Donde unos autores utilizan el producto de $\gamma$ por la masa en reposo $(\gamma \mathrm{m})$, otros emplean la masa relativista, con lo cual no se puede apelar a los resultados experimentales.

Pero actualmente el debate parece cerrarse, porque ha sucedido algo normal en la historia de la física, pero olvidado en su enseñanza: los conceptos se desarrollan, refinan y generalizan en el transcurso del tiempo, es decir, evolucionan. En un momento dado se produce consenso en torno a un concepto (en nuestro caso la masa relativista $m_{r}$ ), después hay discusiones y controversias (Okun, 1989; Sandin, 1991; Bauman, 1994; Bikerstaff y Patsakos, 1995) hasta que se llega a un nuevo consenso. Prueba de ello es el comentario que The European Journal Physics encargó a Okun (1998), titulado «Note on the meaning and terminology of Special Relativity». También se observa que autores en cuyos libros de texto aparecía la masa relativista, en ediciones recientes han modificado esta postura, como, por ejemplo, Alonso y Finn (1995), Tipler (1995), Gettys, Keller y Skove (1992).

Todos ellos se han decantado por el uso de la masa invariante, que coincide con la masa en reposo de una única partícula. Su valor no depende del sistema de referencia elegido (es el escalar invariante asociado al cuadrado del tetravector energía impulso $E^{2}-p^{2} c^{2}=m^{2} c^{4}$ ) y que caracteriza a la partícula o sistema unívocamente.

Se apuntan problemas en del uso de $m_{r}$ como su redundancia con la energía total o el error de sustituir $m$ por $m_{r}$ en ecuaciones clásicas para obtener las relativistas, lo que sólo es correcto en el caso de $p=m_{r} v$ y lleva a expresiones incorrectas en otros casos: $F=m_{r} a$ o $E_{c}=1 / 2 m_{r} v^{2}$. La consideración de la masa relativista como inercial está implícita, en expresiones del tipo: «cuando aumenta la velocidad de un cuerpo, su masa también aumenta» o en la justificación de la velocidad de la luz en el vacío como un valor límite, al indicar que dicha masa aumenta a medida que lo va haciendo la velocidad, de manera que su valor sería infinito cuando la velocidad coincidiera con la de la luz. Al razonar de las formas que se acaban de señalar, se está considerando que la masa relativista ofrece una resistencia a la aceleración. Sin embargo, esta afirmación no es correcta pues $m_{r}$ no resulta ser el coeficiente que relaciona la fuerza y la aceleración.

La perspectiva correcta centra la atención en lo que le ocurre a la partícula en las modificaciones en la estructura del espacio y el tiempo (French, 1991). Esto va hacia lo esencial del planteamiento relativista en cuanto a proceso espaciotemporal y abre paso a los supuestos de la teoría general, en la cual la topología del espacio es protagonista. Por ejemplo, no cabe hablar de efectos gravitacionales sobre la luz sin considerar geodesicas y carecería de sentido atribuir masa a un fotón.

En cuanto a las nociones erróneas de desaparición y aparición de masa en las reacciones nucleares, su extensión e incidencia ha sido puesta de manifiesto en múltiples trabajos (Warren, 1976; Gil et al., 1986; Tarín, 2000). El error suele derivarse de la confusión entre la suma de las masas de las partículas que componen el sistema $\Sigma m_{i}$ y la masa 
del sistema $M$. Surge cuando no se identifica el sistema ni se hace un seguimiento de la evolución del mismo, o no se define correctamente la masa del sistema (invariante relativista). Es claro que $\Sigma m_{i}$ puede cambiar, de hecho lo hace corrientemente en física de partículas o en procesos nucleares y, sin embargo, $M$ permanece constante.

\section{Acerca de la energía}

La teoría de la relatividad completa el cuadro conceptual de la noción de energía trazado a lo largo de toda la enseñanza secundaria mediante la introducción de la equivalencia masa-energía que presenta un expresión nueva para la energía total: $E=\gamma m c^{2}$, que se reduce a $E_{0}=m c^{2}$ en el SRI en reposo. La masa permanece constante en cualquier sistema de referencia, pero el valor de la energía depende del sistema elegido. Éste es el sentido del invariante: $E^{2}-(p c)^{2}=\left(m c^{2}\right)^{2}$ donde para $p=0, E_{0}=m c^{2}$. El razonamiento se extiende de forma natural a los fotones cuya masa es $m=0$ y cuya energía es $E=p c$. Éstas son las relaciones que aplican en su trabajo cotidiano los físicos nucleares o de partículas.

En el tratamiento de los sistemas, por ejemplo, en un gas en reposo, el principio de conservación de la energía conduce a que las eventuales variaciones de energía por intercambio con el exterior, que podría ser un sencillo calentamiento, se acompañen de la paralela variación de masa: $\Delta E=\Delta M c^{2}$. Observemos que es el intercambio con el exterior el que conduce a las variaciones. Es el caso del famoso experimento mental de Einstein, en el cual se emiten dos fotones idénticos en sentidos opuestos. El análisis de las dificultades didácticas de estos problemas conduce a Gil y otros (1986) a recalcar una coherencia profunda: «la identificación de la luz - en la mecánica cuántica- como una forma de existencia de la materia es un resultado totalmente coherente con la ecuación $E=m c^{2}$, que establece que todo intercambio material conlleva un intercambio energético».

En sistemas de partículas en interacción, por ejemplo, las que constituyen un átomo, se pierde la aditividad de la masa entre la del sistema y la de las partículas: $M \neq \Sigma m_{i}$. De forma simple podemos usar como definición de masa de una partícula compleja, un núcleo, por ejemplo: $m=\Sigma m_{i}$ $-E_{b} / c^{2}$ con $E_{b}$ la energía de enlace calculada en el sistema de referencia con $p_{i}=0$.

En procesos que involucran a las partículas, las variaciones en el $\Sigma m_{\text {sistema }}$ son perfectamente posibles, y se traducirán en una variación en la energía cinética de las partículas que componen el sistema. Por ejemplo, en un proceso nuclear $A+B \rightarrow C+D$, la conservación de la energía implica:

$$
Q=\left[\left(m_{D}+m_{C}\right)-\left(m_{A}+m_{B}\right)\right] c^{2}=\left(E c_{B}+E c_{A}\right)-\left(E c_{D}+E c_{C}\right)
$$

En este sentido se habla a veces de conversión de masa en energía (García, 1989). O sea, la conservación de la energía $(\Delta E=0)$ y el cambio en $\Sigma m_{i}$, vienen acompañados por un cambio en la suma de las energías cinéticas, lo que supone una redistribución de energía que, a menudo, se enuncia como transformación, las eventuales alteraciones en las energías de enlace de las partículas se incorporan en la masa invariante de las partículas). Pensemos que, en este caso, las variaciones son de signos opuestos, en contraste con los sistemas abiertos que modifican su energía total $(\Delta E \neq 0)$.

La falta de un adecuado tratamiento didáctico conduce a numerosas confusiones. Parece conveniente resaltar la diferencia entre $M y \Sigma m_{i}$, la inexistencia de «desmaterializaciones», clarificar la diferencia entre energía del sistema y energía cinética presente en el sistema, y su carácter de energía útil, aprovechable para desarrollar trabajo. Es importante indagar acerca del sentido profundo de las «desapariciones de masa», que en todo caso se enmarcan en la conservación de la masa y la energía de los sistemas aislados ( Gil et al., 1986).

El estado actual de la práctica educativa se muestra en un amplio estudio realizado por Tarin (2000), que concluye de forma rotunda: «La equivalencia masa-energía tal y como aparece en la teoría especial de la relatividad no es comprendida por los alumnos. Para algunos de ellos la materia desaparece y se transforma en energía [...] Otros piensan que la energía se encuentra almacenada en la materia y aparece en algunos procesos $[\ldots]$ »

\section{El papel de la historia de la ciencia en la didáctica de la relatividad}

Diversos autores han profundizado en las vinculaciones y paralelismos entre el proceso histórico del nacimiento y la aceptación de las teorías, y el cambio conceptual de los alumnos (Driver et al., 1992, Saltiel y Viennot, 1985, Matthews, 1994). Estas consideraciones cobran una mayor relevancia porque en relatividad se manejan conceptos primarios como espacio, tiempo, simultaneidad, masa... que poseen una rica tradición de controversia histórica muy ilustrativa y paralela a concepciones de los propios alumnos.

\section{DISEÑOS EXPERIMENTALES}

La contrastación de la hipótesis se ha realizado mediante múltiples cuestionarios que revisan el proceso de enseñanza-aprendizaje en la perspectiva de alumnos, profesores y textos.

El análisis de libros de texto, como principal material curricular, se ha realizado en tres niveles: $4^{\circ}$ de ESO, $1^{\circ}$ y $2^{\circ}$ de bachillerato. En los dos primeros niveles se revisará la caracterización clásica del espacio y tiempo, los sistemas de referencia, los fundamentos epistemológicos, la introducción de magnitudes físicas y si se han apuntado limitaciones del marco clásico, lo que permitirá conectar en el siguiente curso con la relatividad. En $2^{\circ}$ de bachiller se revisan: a) aspectos epistemológicos e históricos; $b$ ) aspectos lógicos como la ubicación en el marco de la física, el tratamiento de los principios y las ideas acerca de la propagación de la luz; c) consecuencias clave como las ideas acerca del espacio y del tiempo, la composición de velocidades e ideas sobre la energía y la masa. 
1. $4^{\circ}$ DE ESO Y $1^{\circ}$ DE BACHILLERATO

1.1 ¿Se plantean expresamente los límites de validez de la física newtoniana?

1.2 ¿Se indagan, aun brevemente, los presupuestos de las concepciones newtonianas del espacio y el tiempo?

2. COMPLEMENTO PARA $1^{\circ}$ DE BACHILLERATO

2.1 ¿Se plantea el principio de relatividad de Galileo, discutiendo explícitamente las hipótesis subyacentes?

\begin{tabular}{|c|c|c|}
\hline 3.1 & Se pondera la importancia del experimento de Michelson y Morley. & 46 \\
\hline 3.2 & Se valora adecuadamente el aspecto acumulativo no lineal del avance científico y la existencia de resistencias al cambio. & 27 \\
\hline 3.3 & Se muestran, aunque sea tangencialmente, las repercusiones del avance científico en el entorno cultural. & 36 \\
\hline 3.4 & Se da a entender su aplicación en exclusiva a altas velocidades. & 45 \\
\hline 3.5 & $\begin{array}{l}\text { Se clarifican las posibles ideas alternativas de los alumnos en línea con ideas mantenidas en otros tiempos, } \\
\text { por ejemplo, contracción de Lorentz. Se clarifica la visión microscópica de la contracción. }\end{array}$ & 27 \\
\hline 3.6 & Se muestra la simetría entre las magnitudes en dos sistemas inerciales distintos. & 27 \\
\hline 3.7 & Se clarifica que ver y medir son conceptos diferentes. & 18 \\
\hline 3.8 & Se insiste en la simetría entre las mediciones en SRI distintos. & 27 \\
\hline 3.9 & Se introduce el concepto de masa relativista. & 82 \\
\hline 3.10 & Se reafirma explícitamente la validez del principio de conservación de $P$ en un $S R I$ así como la de otras leyes de la física. & 36 \\
\hline
\end{tabular}

En los cuestionarios de alumnos y profesores se manejan aspectos análogos para revisar la coherencia entre los resultados obtenidos. Con los profesores se apunta al tratamiento epistemológico que proponen y la forma de introducir conceptos y sus actitudes. Con los alumnos se revisa la naturaleza del aprendizaje que efectúan y el punto de partida con que se inicia el proceso. Se muestra una selección de los cuestionarios junto con las tablas de resultados.

\section{RESULTADOS DEL ESTUDIO DE LIBROS DE TEXTO}

Se ha realizado un análisis de 30 libros de texto de reciente edición, 7 de $4^{\circ}$ de ESO, 12 de $1^{\circ}$ de bachillerato y 11 de $2^{\circ}$ de bachillerato. Los resultados se presentan a continuación en forma de porcentaje de respuestas afirmativas a cada cuestión.

En la cuestión 3.1 podemos apreciar una deformación corriente: la sobrevaloración del papel jugado por el experimento de Michelson y Morley. También en el análisis de su repercusión aparecen distorsiones como un empirismo que plantea, en primer lugar, el experimento y, a continuación, la teoría, minusvalorando la inventiva que condujo a la teoría de la relatividad (Holton, 1982),
No se patentizan los propios límites de la relatividad especial, se induce con ello la idea de la ciencia como un conocimiento acabado. Incluso los relevantes aspectos CTS sólo se tratan como muestran los resultados del ítem 3.2, en el $36 \%$ de los textos.

Todavía aparecen opciones hoy superadas, tales como el uso de la masa relativista. En los textos de $1^{\circ}$ de bachillerato, la masa, en general, no merece una excesiva atención. Nos encontramos desde la mera omisión de una definición de la misma, a la definición tradicional de masa como cantidad de materia del cuerpo que aparece en cuatro de los textos, en frases como: «La masa inerte es la cantidad de materia del cuerpo». Incluso en dos textos de $1^{\circ}$ se avanza presentando la masa relativista, lo que contribuye a reforzar la hipótesis de que su uso está todavía bastante extendido entre los materiales didácticos.

En $2^{\circ}$, un $82 \%$ de los textos analizados hace referencia al concepto de masa relativista (ítem 3.9). Estos resultados son coherentes con los obtenidos por Sánchez (2000). Cabe matizar en cuanto a la importancia atribuida al concepto. Algunos se enmarcan totalmente en el uso de la $m_{r}$, por ejemplo: «Einstein dedujo a partir del principio de conservación de la cantidad de movimiento que la masa de un cuerpo depende de su velocidad según la siguiente fórmula: $m=\left(1 / \sqrt{ } 1-v^{2} / c^{2}\right) m_{0} \ldots$ Se observa que la masa de un cuerpo aumenta con su velocidad, de tal manera que, cuando 
su velocidad se aproxima mucho a $c$, la masa se hace infinitamente grande. Esto significa que la fuerza necesaria para acelerar un cuerpo hasta la velocidad de la luz es infinita $[\ldots] \gg$. En este extracto se condensan muchos puntos de interés: hay una deformación acerca del papel de Einstein (él no la introdujo) y se observa cómo ha operado en el autor el mecanismo erróneo de utilizar esta masa relativista inercialmente al razonar en torno a la fuerza necesaria para acelerar la partícula y acerca de la existencia de una velocidad límite. Esto se da en otros dos textos, en que se ha usado de forma errónea $F=m_{r}$. a, como ampliación de la ley clásica. Estos mecanismos mentales son los que didácticamente se desea evitar en los estudiantes.

Otros textos la asumen de un modo convencional y no atribuyen a la masa relativista un carácter físico esencial sino simplemente formal, como agrupación funcional de magnitudes.

En cuanto a la equivalencia masa-energía en dos de los textos se realizan afirmaciones erróneas, manejando de forma inapropiada conceptos tales como la conversión masa-energía. Frases del tipo «la suma de la masa y energía del sistema ha de permanecer constante» no se corresponden con lo que se ha debatido anteriormente. Admitirlas supondría soslayar la gran aportación de Einstein de energía asociada a la masa en reposo. Además de suponer la invalidez del principio de conservación de la energía, parece como si la cantidad de energía presente en un sistema cerrado dependiera de eventuales desapariciones de masa en el interior del sistema, en la cantidad $E / c^{2}$.

Respecto al tiempo, se observa en algún texto que se manejan dos planos diferentes de realidad, según sea el tiempo propio o el correspondiente a un fenómeno que sucede en un cuerpo en movimiento. Para este caso se reserva la palabra aparente, lo que viene a negar la propia relatividad. Todos los tiempos evaluados en un sistema de referencia son igualmente reales en ese sistema. No son admisibles expresiones como: «el corazón del pasajero aparentemente late más despacio», «el tiempo de un sistema en movimiento parece dilatarse respecto al tiempo medido en un sistema en reposo solidario con el observador».

Por último, tampoco parece establecido un acuerdo tácito entre los autores en torno al nivel de complejidad correspondiente a $2^{\circ}$ de bachillerato. La dispersión entre temas así como la extensión y profundidad de los tratamientos es muy amplia, probablemente debido a la escasa tradición de su enseñanza en el bachillerato Por ejemplo, hay quien introduce espacios de Minkowski, la noción de intervalo manejando números complejos. Algunos apuntan nociones de la teoría general y otros no la mencionan...

\section{RESULTADOS DEL CUESTIONARIO DE PROFESORES}

Se ha obtenido la colaboración de 37 profesores en activo $\mathrm{y}$ formación sobre aspectos epistemológicos, históricos y sobre la introducción de diversos conceptos.
1.1. ¿Qué aspectos señalarías como más significativos, en el desarrollo histórico de la relatividad especial y que, a tu juicio, deberían presentarse a los estudiantes?

La referencia a una situación problemática solamente se encuentra presente en un $30 \%$ de las respuestas, pero sin pasar de una mención genérica sobre la necesidad de tomar en consideración los antecedentes previos. En tan sólo en un $15 \%$ de las respuestas se hace referencia directa al proceso de evolución de la física moderna versus la física clásica.

Entre las respuestas anteriores (un total de 45\%) se incluye un $28 \%$ de quienes resaltan el proceso de elaboración de la teoría especial por Einstein, pero, por lo general, sin una elaboración que sugiera una epistemología o conocimiento suficiente del proceso histórico. Se suele limitar a señalar que se debería suministrar datos acerca de Einstein y su vida.

Las reflexiones sobre la acogida por la comunidad científica de la TER aparecen también en un tercio de los casos, como la "validación por la comunidad científica». Sin embargo esto no pasa de ser una percepción genérica de resistencia al cambio, por ejemplo: «[...] la época en la cual Einstein formuló la teoría 1905, en la cual la sociedad le criticó $[\ldots] \gg$

2.1. Indica qué aspectos de relatividad convendría introducir y en qué orden. Justificalo brevemente. Indica, en cada caso, si se propone una introducción cualitativa o cuantitativa.

En el conjunto de la muestra, únicamente el 5\% de las respuestas propone una estrategia coherente con un hilo conductor claro para la introducción a la teoría de la relatividad. En cuanto al núcleo central de la teoría, los elementos esenciales del principio de relatividad o su estatus en la física están escasamente representados, y el estudio de los sistemas de referencia y el propio principio de relatividad no aparecen sino en un $13 \%$ de las respuestas. Priman las deducciones más habituales que se derivan de los postulados: la configuración del espacio-tiempo, en su faceta más llamativa de contracciones y dilataciones, sin excesiva profundización es el concepto dominante.

Por otra parte, están presentes expresiones y frases que revelan un desconocimiento profundo de los aspectos más básicos de la teoría; por ejemplo, algunas respuestas indican que no se diferencia la relatividad einsteniana de la clásica. Son respuestas del tipo: «Permite hacer distinción entre un hombre que ve pasar un tren y el que viaja dentro» o relacionarla con explicaciones del «movimiento de los planetas...».

2.3. La ecuación $E=m c^{2}$ es una de las más conocidas y divulgadas. ¿Cómo la explicarías a los alumnos para intentar que capten su significado? 
En el manejo de $E=m c^{2}$ se encuentran expresiones directas de interconversión (17\%) del tipo «la masa de cualquier cuerpo tiene la capacidad potencial de transformarse en energía». Se prescinde así del concepto de energía asociada a la masa invariante $E_{0}=m c^{2}$, y viceversa. La noción errónea que se mantiene es similar a una «desmaterialización de la materia» y análoga al concepto erróneo sobre la combustión difundido en ocasiones entre los alumnos, quienes lo interpretan como una desaparición de materia.

La interpretación de la ecuación en términos de equivalencia se da en un $21 \%$ de respuestas; sin embargo; la interpretación de la ecuación no es unívoca. Hay quien identifica totalmente masa y energía, «son lo mismo», y quien las diferencia: «todo cuerpo por tener masa tiene energía asociada». La igualdad entre ambos conceptos ya se ha desechado anteriormente; por ejemplo, un fotón tiene energía y, sin embargo, se considera una partícula sin masa. La equivalencia la planteamos en los términos en que la planteó Einstein en 1905: la existencia de una energía correspondiente a una masa en reposo $\left(E_{0}=m c^{2}\right)$.

Un $54 \%$ plantea la relación como una vinculación similar a la relación entre la masa y el volumen, con una separación profunda entre los conceptos. En esta categoría se engloban las respuestas que plantean una relación de dependencia funcional entre magnitudes independientes (sintetizada en una fórmula) análoga al de la $E$ cinética clásica. Este uso meramente funcional origina propuestas de estrategia de aprendizaje mediante un mero operativismo: «la explicación detallada de las distintas magnitudes que intervienen en la fórmula».

En otras, la respuesta no proporciona una explicación acerca de la equivalencia (incluyendo el $8 \%$ con deformaciones importantes).

\section{RESULTADOS OBTENIDOS EN EL CUESTIO- NARIO DE ALUMNOS}

Se muestran a continuación los resultados obtenidos de los cuestionarios pasados a los estudiantes, que se han obtenido en contextos diferentes y con un perfil distinto de los alumnos: 21 de $4^{\circ}$ de ESO, 80 de $1^{\circ}$ de bachillerato, 54 de $2^{\circ}$. Se muestra a continuación una selección de ítems y resultados con sus comentarios.

\begin{tabular}{|l|l|c|c|c|}
\hline $1^{\circ}$ & \multicolumn{4}{|c|}{ Expón las ideas que tengas sobre el espacio. ¿Qué es el espacio? } \\
\hline $\begin{array}{l}\text { Manifiesta una concepción } \\
\text { apropiada del espacio, } \\
\text { mínimamente diferenciado } \\
\text { en características y atributos. }\end{array}$ & $4^{\circ}$ ESO & $1^{\circ}$ bach. & $2^{\circ}$ bach. \\
$\begin{array}{l}\text { Ajustada al nivel: } \\
\text { clásico } 4^{\circ} / 1^{\circ}, \text { relativista } \\
\text { en } 2^{\circ} \text { bachillerato) }\end{array}$ & & $19 \%$ & $13 \%$ \\
\hline
\end{tabular}

Únicamente un pequeño porcentaje de alumnos de ESO y $1^{\circ}$ de bachillerato posee un concepto clásico del espacio dotado de cierta entidad y enuncia, al menos, un par de atributos del mismo. Se observa poca diferencia entre los alumnos de estos cursos y los de $2^{\circ}$, pese a que estos últimos han realizado un estudio mucho más amplio de mecánica.

Un estudio más detenido de las respuestas muestra la forma en que lo definen y las propiedades que le atribuyen. La concepción de los estudiantes de $4^{\circ}$ de ESO y $1^{\circ}$ de bachillerato asemeja el espacio a un contenedor, escenario de los fenómenos, pasivo, que no interacciona con ellos; pero parece muy poco madurada y se puede inferir que no ha sido objeto de reflexión. De hecho, parece que en $1^{\circ}$ de bachillerato sólo se ha manejado el término espacio en contextos cinemáticos. La base de la explicación es intuitiva, matizada en ocasiones por conocimientos dispersos acerca de la estructura del universo.

Paradójicamente, los estudiantes de $2^{\circ}$ de bachillerato no fijan su atención en estas propiedades y se dedican a resaltar el carácter no universal del espacio y su dependencia con el sistema de referencia. Esto es indicativo de que el aprendizaje se orienta a resaltar la diferencia con las concepciones clásicas sin un estudio de la situación problemática de partida ni profundizar en los conceptos.

\begin{tabular}{|c|c|c|c|c|}
\hline $2^{\circ}$ & \multicolumn{4}{|c|}{ Expón las ideas que tengas sobre el tiempo. } \\
\hline & $\begin{array}{l}\text { lifiesta una concepción } \\
\text { piada del tiempo, } \\
\text { imamente diferenciado } \\
\text { aracterísticas y atributos. } \\
\text { istada al nivel: } \\
\text { ico } 4^{\circ} / 1^{\circ}, \text { relativista en } \\
\text { achillerato) }\end{array}$ & $\begin{array}{c}4^{\circ} \mathrm{ESO} \\
15 \%\end{array}$ & $\begin{array}{c}1^{\circ} \text { bach. } \\
12 \%\end{array}$ & $\begin{array}{c}2^{\circ} \text { bach. } \\
20 \%\end{array}$ \\
\hline
\end{tabular}

El concepto de tiempo presenta una mayor dificultad de verbalización, lo que se traduce en menores porcentajes de explicaciones suficientemente correctas. Los estudiantes de $4^{\circ}$ de ESO y $1^{\circ}$ de bachillerato no recogen un abanico amplio de propiedades en la perspectiva clásica. El tiempo clásico se entiende como un parámetro real infinito $(5 \%$ en $\left.1^{\circ}\right)$, monótonamente creciente $\left(5 \%\right.$ en $4^{\circ}$ de ESO, $3 \%$ en $1^{\circ}$ ), continuo $\left(5 \%\right.$ en $4^{\circ}, 3 \%$ en $\left.1^{\circ}\right)$, homogéneo, isótropo, universal $\left(10 \%\right.$ en $\left.4^{\circ}\right)$, pasivo e independiente del espacio. Esta gama de propiedades no se cita más que en un porcentaje mínimo.

En $2^{\circ}$, el abanico de propiedades paradójicamente se estrecha, pues se centran en exclusiva en el aspecto relativo del tiempo y se empobrece, incluso más, la presencia de otras propiedades; valoran en cambio mucho las relaciones de Lorentz, que ilustran muchas respuestas. El 61\% son capaces de referirse al carácter relativo, no universal del tiempo, diferente según el observador. El aspecto más señalado en $2^{\circ}$ es la mención a la evolución desde las nociones clásicas a la relativista pero sin concretar el concepto.

Predominan las imágenes del tiempo como duración de fenómenos, con movimientos periódicos, etc. y con muy escaso dominio de sus propiedades, lo que apunta a lo superficial del trabajo realizado en torno al concepto. 


\begin{tabular}{|l|l|l|l|}
\hline $3^{\circ}$ & \multicolumn{3}{|c|}{$\begin{array}{l}\text { ¿Existen sistemas de referencia que estén totalmente } \\
\text { quietos, en reposo absoluto? ¿Cuáles? }\end{array}$} \\
\hline $\begin{array}{l}\text { Manifiesta una concepción } \\
\text { apropiada del concepto } \\
\text { de espacio absoluto y rechazan } \\
\text { con argumentos su existencia. }\end{array}$ & $\begin{array}{c}4^{\circ} \text { ESO } \\
0 \%\end{array}$ & $\begin{array}{c}1^{\circ} \text { bach. } \\
7 \%\end{array}$ & $\begin{array}{c}2^{\circ} \text { bach. } \\
9 \%\end{array}$ \\
\hline
\end{tabular}

El concepto de espacio absoluto no parece ser plenamente comprendido, pues, a pesar de usar varios sistemas de referencia y comprender la relatividad del movimiento, todo parece discurrir para ellos en un marco que es la auténtica realidad del espacio.

Un porcentaje muy bajo de estudiantes de $2^{\circ}$ de bachillerato $(9 \%)$ parece haber efectuado el tránsito hacia la comprensión de este espacio. Si se toma en consideración aquellas respuestas que muestran razonadamente que queda superado por la teoría de la relatividad, este porcentaje bajaría todavía más.

\begin{tabular}{|c|l|c|}
\hline $4^{\mathrm{o}}$ & $\begin{array}{l}\text { Indica, a modo de resumen, los que consideres } \\
\text { puntos esenciales en la relatividad. }\end{array}$ & \\
\cline { 2 - 3 } & $\begin{array}{l}\text { Expone efectivamente los puntos esenciales } \\
\text { de la relatividad especial }\end{array}$ & $\begin{array}{c}2^{\circ} \mathrm{bach} . \\
32 \%\end{array}$ \\
\hline
\end{tabular}

En las respuestas se da una dispersión, un 30\% de los estudiantes no es capaz de indicar algún punto esencial o no se centra en ningún aspecto relevante, otro $26 \%$ destaca como aspecto más relevante el uso de masa relativista. Otro 7\% indica como tal la equivalencia masa-energía.

Entre los puntos más señalados por los estudiantes se indican, por ejemplo, la velocidad de la luz como límite físico (esencial para el 22\%). De hecho, el punto central del principio de relatividad es citado por el $24 \%$ de las respuestas, porcentaje sumamente bajo. Las nociones básicas de espacio se mencionan con una explicación acerca de su papel (37\%) y el concepto de simultaneidad y la nueva concepción del tiempo (31\%).

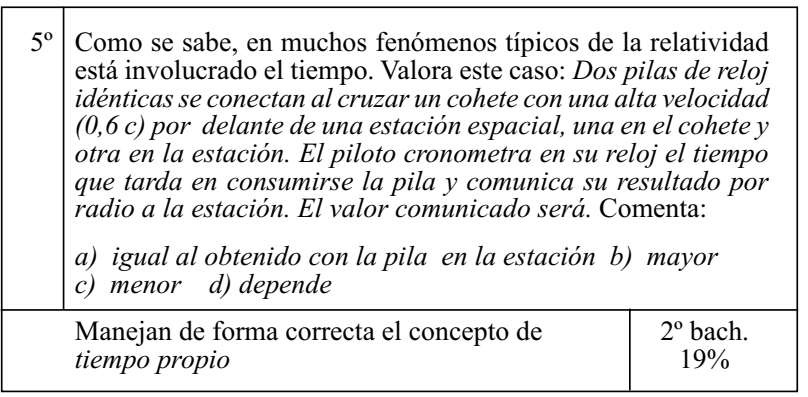

Hay un reparto equilibrado entre las respuestas de mayor $(37 \%)$ y menor duración $(40 \%)$. El tipo de respuestas incorrectas son, por ejemplo: «En la pila que ha viajado a una velocidad cercana a la de la luz, el tiempo ha transcu- rrido más despacio, se produce lo que llamamos una dilatación del tiempo.» El estudiante se ha dejado arrastrar por la medición efectuada desde un sistema sobre el otro y no por la medición que realiza el otro sistema del fenómeno, que no puede sino ser idéntica a la suya propia, so pena de privilegiar uno sobre otro. Se detecta también una importante presencia de la expresión $\Delta t^{\prime}=\Delta t /\left(1-v^{2} / c^{2}\right)^{1 / 2}$, escrita en el $37 \%$ de los casos, utilizada en un sentido u otro para argumentar tiempos mayores o menores. Se incide en un operativismo que no es de aplicación al caso.

En suma, se puede considerar que el aprendizaje realizado por la generalidad de los alumnos dista de ser suficientemente significativo conforme se propone en la hipótesis.

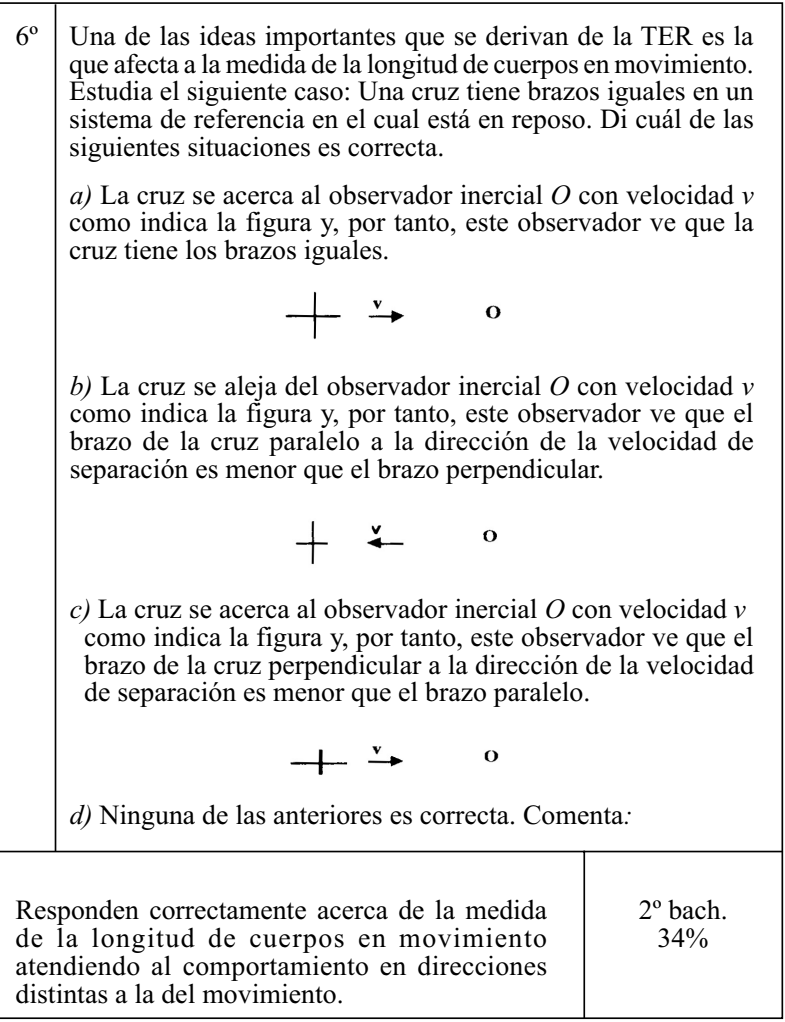

Hay un porcentaje sustancial de respuestas que no aciertan a diferenciar las transformaciones geométricas cuando se solicita evaluar longitudes en situaciones no evidentes, lo cual es indicativo de que no se ha insistido en la asimetría entre las direcciones, ni el hecho de que es indiferente si se trata de un acercamiento o alejamiento en una dirección.

\begin{tabular}{|c|c|c|}
\hline $7^{\circ}$ & $\begin{array}{l}\text { Las fórmulas pueden ser difíciles, uno de los trucos que se } \\
\text { utiliza a veces es aprovechar las ecuaciones conocidas de la } \\
\text { mecánica sustituyendo } m \text { por } m_{0} /\left(1-v^{2} / c^{2}\right)^{-1 / 2} \text {. ¿Es, en tu } \\
\text { opinión, una buena estrategia o puede llevar a error en algún } \\
\text { caso? }\end{array}$ \\
\hline $\begin{array}{l}\text { Proporciona una opinión correcta acerca de lo } \\
\text { inadecuado de la sustitución en las expresiones } \\
\text { dinámicas. }\end{array}$ & $2^{\circ}$ bach. \\
$15 \%$
\end{tabular}


El porcentaje de alumnos que identifican problemas en la extensión de $m_{r}$ a otras de fórmulas de forma indiscriminada es muy pequeño (15\%). Abundan, por el contrario, posiciones erróneas e incluso son más numerosas las que no se definen (44\%). Un 41\% traslada directamente la fórmula a las expresiones clásicas, lo que es indicativo de que constituye una fuente sustancial de error e indirectamente favorece nuestra hipótesis.

\begin{tabular}{|c|l|c|}
\hline $8^{\circ}$ & $\begin{array}{l}\text { La ecuación } E=m c^{2} \text { es una de las más conocidas. } \\
\text { ¿Cuál es su significado? }\end{array}$ \\
\hline $\begin{array}{l}\text { Proporciona una explicación correcta del significado } \\
\text { de la ecuación } E=m c^{2}, \text { manejando la masa invariante. }\end{array}$ & $\begin{array}{c}2^{\circ} \mathrm{bach} . \\
19 \%\end{array}$ \\
\hline
\end{tabular}

Un $19 \%$ asume la masa invariante $m$ manejando $E_{0}=m c^{2}$ y siguen una interpretación basada en la equivalencia energía y masa (en reposo). Una mayoría (41\%) interpreta la ecuación usando $m_{r}$. Se dan otras respuestas que se limitan a la lectura funcional de la fórmula análoga al de la energía cinética clásica, sin idea de equivalencia, que sería arriesgado encuadrar en alguna de las otras categorías y que apuntan hacia una inferior comprensión. Podemos ilustrar estas posiciones con respuestas del tipo: «que la energía es directamente proporcional a la masa y que, si hiciéramos una gráfica, variarían linealmente», simple trascripción de la fórmula analítica.

\begin{tabular}{|c|l|c|}
\hline $9^{\circ}$ & $\begin{array}{l}\text { Cuando un núcleo de uranio } 235 \text { se rompe en dos o más } \\
\text { fragmentos, se libera una energía de } 200 \mathrm{MeV} \text { por fisión. } \\
\text { Explica este hecho. }\end{array}$ \\
\hline $\begin{array}{l}\text { Razona correctamente acerca de la energía } \\
\text { de los procesos nucleares de fisión }\end{array}$ & $\begin{array}{c}2^{\circ} \mathrm{bach} . \\
22 \%\end{array}$ \\
\hline
\end{tabular}

Los razonamientos energéticos prácticos son en general bastante confusos y tan sólo un $22 \%$ se puede conceptuar como suficientemente correcto, lo que indica las dificultades que presenta este punto.

- Las explicaciones usan razonamientos de interconversión masa-energía ( $15 \%$ de respuestas) del tipo: «energía aumenta cuando la masa disminuye, por tanto, la igualdad se conserva. La pérdida de esta materia es la que nos aporta dicha energía». La línea argumental es análoga a la considerada en el caso del ítem 2.1 de profesores: prescinden de la energía asociada a la masa en reposo, identifican energía con energía cinética, violan el principio de conservación de la energía en sistemas aislados, etc. En algún caso apelan incluso al principio de conservación: «esto se debe al principio de conservación de la energía». Sin embargo, estas apelaciones, encubren una falta de explicación acerca del origen de la energía:

- Manejan esquemas o modelos mecánicos incorrectos $(11 \%)$. Hay explicaciones que ubican la energía según esquemas mentales de recipiente, la energía sería un tipo de ente sin substrato que se libera al romperse el átomo: «Lo que ocurre es que el uranio, debido a su masa, tiene una gran energía interna según la ecuación $E=m c^{2}$ y al romperse libera toda esa energía que tenía en su interior 200 $M e V »$. En esa línea se orientan otras respuestas: «Debido al principio de conservación de la energía el núcleo de uranio tiene energía interna que al realizar en él una fisión sale al exterior».

- No comprenden la variación de masa que se produce (7\% de las respuestas). Por ejemplo, algunos la achacan a la fisión: "Cuando se rompe la masa se divide en dos y, por lo tanto, pierde masa, ya que la masa total se reparte en dos.» No proporcionan una respuesta o es errática en el $30 \%$ de los casos, lo que no les ocurre en otras cuestiones.

\section{CONCLUSIONES Y PERSPECTIVAS}

Del estudio de los instrumentos desarrollados podemos fijar las siguientes conclusiones:

1) Los libros de texto utilizados en los niveles inferiores de la secundaria que se han analizado no presentan adecuadamente los conceptos de tiempo y espacio. En $2^{\circ}$ de bachillerato, la enseñanza de la teoría de la relatividad se plantea de forma poco clarificadora, sin tener en cuenta las preconcepciones de los alumnos y sin resaltar su posición en la estructura de la física. Se apoya esta afirmación en datos tales como que el estudio del principio de relatividad galileano no se realiza en más del $80 \%$ de los textos de primer nivel, o que en los textos de $2^{\circ}$ de bachillerato se da una importante presencia de la masa relativista (en el $82 \%$ de los textos) y muy diversos y contradictorios enfoques sobre su significado.

2) En la práctica habitual los profesores encuestados introducen, de forma acrítica y poco reflexiva los conceptos, desde orientaciones epistemológicas distorsionadas y sin contar con los resultados de la investigación didáctica. Estas conclusiones se deducen del estudio de las respuestas de los profesores que sólo plantean la necesidad de partir de una situación problemática en el $30 \%$ de los casos, con dificultades de entresacar lo sustancial de la teoría, manejar aplicaciones de la relatividad o razonar adecuadamente sobre la masa, incluso con un $17 \%$ que razona en términos de conversión masa-energía, etc.

3) Los alumnos, como consecuencia de la enseñanza recibida, muestran un aprendizaje escasamente significativo y se consolidan poco las nuevas concepciones en los estudiantes. Esta conclusión se extrae directamente de la escasa comprensión sobre conceptos básicos, por ejemplo, una concepción correcta del espacio, en la perspectiva relativista, no es alcanzada por más del $13 \%$ de los estudiantes; el manejo de conceptos como el de tiempo propio no se realiza correctamente por el $80 \%$ de los estudiantes; un $68 \%$ no señala los puntos esenciales de la relatividad; o el $78 \%$ no es capaz de razonar adecuadamente acerca de la energía en procesos tales como la fisión nuclear.

Justificada de esa forma la utilidad de proseguir este estudio, cabe iniciar la tarea por una ampliación de los instru- 
mentos (por ejemplo, con entrevistas, otros cuestionarios, etc.) y por incrementar el tamaño de las muestras. También se planteará una segunda fase que consista en la elaboración de una propuesta alternativa que incida en los puntos puestos de manifiesto en este trabajo y en línea con el proceso de enseñanza por investigación, que se aplicará a otros alumnos, comparando los resultados. Por último, puede ser interesante indagar en los primeros niveles de la enseñanza universitaria, lo que permitiría profundizar en aspectos cuya complejidad y extensión exige una mayor madurez y que adicionalmente iluminarían estrategias transferibles hacia la secundaria.

\section{REFERENCIAS BIBLIOGRÁFICAS}

ADLER, C.G. (1987). Does mass really depend on velocity, dad? American Journal of Physics, 55(8) pp. 739-743.

ALEMAÑ, R.A. (2000). Enseñanza por cambio conceptual: De la física clásica a la relatividad. Enseñanza de las Ciencias, 18(3), pp. 463-471.

ALONSO, M. y FINN, E.J. (1995). Física. Wilmington: Addison Wesley.

BAUMAN, R.P. (1994). Mass and Energy: The Lower-Energy limit. The Physics Teacher, 32, pp. 340-342.

BICKERSTAFF, R.P. y PATSAKOS, G. (1995). Relativity generalizations of mass. European Journal of Physics, 16, pp. 63-66.

CARSON, S. (1998). Relativistic mass. Physics Education, 33(6) pp. 343-345

COLOMBO DE CUDMANI, L. (1995). Distintos tipos de constantes en física y aprendizaje significativo de la disciplina. Enseñanza de las Ciencias, 13(2), pp. 237-248.

DRIVER, R., GUESNE, E. y TIBERGHIEN, A. (1992). Ideas científicas en la infancia y la adolescencia. Madrid: Morata MEC.

FRENCH, A.P. (1991). Relatividad especial. Barcelona: Reverte.

GALLEGOS, J.A. (1992). Errores conceptuales en geología: los conceptos de isotropía, anisotropía y propiedad escalar propiedad vectorial. Enseñanza de las Ciencias, 10(2), pp. 159164.

GARCÍA, G. (1989). Sobre masa y energía. Revista Española de Física, 3, pp. 59-62.
GETTYS, W.E., KELLER, F.J. y SKOVE, M.J. (1993). Física. Madrid: McGraw-Hill.

GIL, D. y SOLBES, J. (1993). The introduction of modern physics: overcoming a deformed vision of science. International Journal of Science Education, 15(3), pp. 255-260.

GIL, D., SENENT, F. y SOLBES, J. (1986). Análisis crítico de la introducción de la física moderna en la enseñanza media. Revista de Enseñanza de la Física, 2, pp. 16-21.

HEWSON, P.W. (1982). A case study of conceptual change in special relativity: The influence of prior knowledge in learning. European Journal of Research in Science Education, 4(1), pp. 61-78.

HIERREZUELO, J. (1993). Ciencias de la naturaleza: física y química. Educación secundaria $3^{\circ}$ y $4^{\circ}$. Comentarios. VélezMálaga: Elzevir.

HOLTON, G. (1982). Einstein, Michelson y el experimento crucial, en Ensayos sobre el pensamiento científico en la época de Einstein, pp. 204-294. Alianza: Madrid.

MATTHEWS, M. (1994). Historia, filosofía y enseñanza de las ciencias: La aproximación actual. Enseñanza de las Ciencias, 12(2), pp. 255-277.

OKUN, L.B. (1989). The concept of mass. Physics Today, 42, pp. 31-36.

OKUN, L.B. (1998). Note on the meaning and terminology of Special Relativity. European Journal of Physics, 15, pp. 403406.

OSTERMANN, F. y MOREIRA M.A. (2000). Física contemporánea en la escuela secundaria: una experiencia en 
el aula involucrando formación de profesores. Enseñanza de las Ciencias, 18(3), pp. 391-400.

PARASNIS, A.S. (1998). Motion, Matter, Mass, Laws of Motion, Newton and Einstein. Physics Education. July-September, pp. 109-116.

ROMERO, C. (1996). Una investigación sobre los esquemas conceptuales del continuo. Enseñanza de las Ciencias, 14(1) pp. 1-14.

SALTIEL, E. y MALGRANGE, J.L. (1980). Spontaneous ways of reasoning in elementary kinematics. European Journal of Physics, 2, pp. 73-80.

SALTIEL, E. y VIENNOT, L. (1985). ¿Qué aprendemos de las semejanzas entre las ideas históricas y el razonamiento espontáneo de los estudiantes? Enseñanza de las Ciencias, 3, pp. 137-144.

SÁNCHEZ, J.L. (2000). El concepto relativista de masa inerte en los textos de física del nuevo bachillerato. Revista Española de Física, 14(4).

SÁNCHEZ RON, J.M. (1985). El origen y el desarrollo de la relatividad, p. 63. Madrid: Alianza.

SANDIN, T.R. (1991). In defense of relativistic mass. American Journal of Physics, 59(11), pp. 1032-1036.
SOLBES, J., BOTELLA, F., PÉREZ, H. y TARÍN, F. (2002). Algunas consideraciones sobre la masa (o masa no hay más que una). Revista Española de Física, 16(1), pp. 47-51.

SHABAJEE, P. y POSTLETHWAITE, K. (2000). What happened to modern physics. School Science Review, 81(297) pp. 51-55.

TARÍN, F. (2000). «El principio de conservación de la energía y sus implicaciones didácticas». Tesis doctoral. Universitat de València.

TIPLER, P.A. (1995). Física. Barcelona: Reverté.

TOLEDO, B., ARRIASSECO, I. y SANTOS, G. (1997). Análisis de la transición de la física clásica a la relativista desde la perspectiva del cambio conceptual. Enseñanza de las Ciencias, 15(1), pp. 79-80.

VILLANI, A. y PACCA, J.L.A. (1987). Students spontaneous ideas about the speed of light. International Journal of Science Education, 1, pp. 55-66.

VILLANI, A. y ARRUDA, S. (1998). Special Theory of Relativity, conceptual change and history of science. Science \& Education, 7(1), pp. 85-100.

WARREN, J.W. (1976). The mistery of mass-energy. Physics Education, 11, pp. 52-54.

WILLIAMS, P. (1968). La teoría de la relatividad. Madrid: Alianza.

[Artículo recibido en diciembre de 2001 y aceptado en octubre de 2002.] 